\title{
Riemann Boundary Value Problem for Triharmonic Equation in Higher Space
}

\author{
Longfei Gu \\ Department of Mathematics, Linyi University, Linyi, Shandong 276000, China \\ Correspondence should be addressed to Longfei Gu; longfei-gu@whu.edu.cn \\ Received 18 January 2014; Revised 19 June 2014; Accepted 19 June 2014; Published 8 July 2014 \\ Academic Editor: Argiris I. Delis
}

Copyright ( 2014 Longfei Gu. This is an open access article distributed under the Creative Commons Attribution License, which permits unrestricted use, distribution, and reproduction in any medium, provided the original work is properly cited.

We mainly deal with the boundary value problem for triharmonic function with value in a universal Clifford algebra: $\Delta^{3}[u](x)=0$, $x \in \mathscr{R}^{n} \backslash \partial \Omega, u^{+}(x)=u^{-}(x) G(x)+g(x), x \in \partial \Omega,\left(D^{j} u\right)^{+}(x)=\left(D^{j} u\right)^{-}(x) A_{j}+f_{j}(x), x \in \partial \Omega, u(\infty)=0$, where $(j=1, \ldots, 5) \partial \Omega$ is a Lyapunov surface in $\mathscr{R}^{n}, D=\sum_{k=1}^{n} e_{k}\left(\partial / \partial x_{k}\right)$ is the Dirac operator, and $u(x)=\sum_{A} e_{A} u_{A}(x)$ are unknown functions with values in a universal Clifford algebra $\mathrm{Cl}\left(V_{n, n}\right)$. Under some hypotheses, it is proved that the boundary value problem has a unique solution.

\section{Introduction and Preliminaries}

The theory of Riemann boundary value problems in complex plane has been systematically developed in $[1,2]$. It is an interesting topic to generalize the classical Riemann boundary value problems theory to Clifford analysis. In [3-6], and so forth, many interesting results about boundary value problem and Riemann Hilbert problems for monogenic functions in Clifford analysis are presented. In [7], Green's function for the Dirichlet problem for polyharmonic equations was studied. The aim of this paper is to study the Riemann boundary value problem for triharmonic functions. At first, based on the higher order Cauchy integral representation formulas in $[8,9]$ and the Plemelj formula, we give some properties of triharmonic functions in Clifford analysis, for example, the mean value theorem, the Painleve theorem, and so forth. Furthermore, on the basis of the above results, we consider the following Riemann boundary value problems:

$$
\begin{gathered}
\Delta^{3}[u](x)=0, \quad x \in \mathscr{R}^{n} \backslash \partial \Omega, \\
u^{+}(x)=u^{-}(x) A+g(x), \quad x \in \partial \Omega, \\
\left(D^{j} u\right)^{+}(x)=\left(D^{j} u\right)^{-}(x) A_{j}+f_{j}(x), \quad x \in \partial \Omega, \\
|u(\infty)| \leq C^{*},
\end{gathered}
$$

$$
\begin{gathered}
\Delta^{3}[u](x)=0, \quad x \in \mathscr{R}^{n} \backslash \partial \Omega, \\
u^{+}(x)=u^{-}(x) G(x)+g(x), \quad x \in \partial \Omega, \\
\left(D^{j} u\right)^{+}(x)=\left(D^{j} u\right)^{-}(x) A_{j}+f_{j}(x), \quad x \in \partial \Omega, \\
u(\infty)=0,
\end{gathered}
$$

where $(j=1, \ldots, 5)$.

In (1) and (2), $A$ and $A_{j}$ are invertible constants; we denote the inverse elements as $A^{-1}$ and $A_{j}^{-1} \cdot u(x),\left(D^{j} u\right)(x)$, $g(x), f_{j}(x) \in H^{\beta}\left(\partial \Omega, \mathrm{Cl}\left(V_{n, n}\right)\right), j=1, \ldots, 5,0<\beta \leq 1$. The explicit solutions for (1) are given and the boundary value problem (2) is shown to have a unique solution under some hypotheses.

Let $V_{n, s}(0 \leq s \leq n)$ be an $n$-dimensional $(n \geq 1)$ real linear space with basis $\left\{e_{1}, e_{2}, \ldots, e_{n}\right\}$, and $\mathrm{Cl}\left(V_{n, s}\right)$ the universal Clifford algebra over $V_{n, s}$. For more information on $\mathrm{Cl}\left(V_{n, s}\right)(0 \leq s \leq n)$, we refer to [10-12].

Throughout this paper, suppose $\Omega$ is an open, bounded nonempty subset of $\mathscr{R}^{n}$ with a Lyapunov boundary $\partial \Omega$, denoting $\Omega^{+}=\Omega, \Omega^{-}=\mathscr{R}^{n} \backslash \bar{\Omega}$. In this paper, for simplicity, we will only consider the case of $s=n$. The operator $D$ is given as

$$
D=\sum_{k=1}^{n} e_{k} \frac{\partial}{\partial x_{k}}: C^{r}\left(\Omega, \mathrm{Cl}\left(V_{n, n}\right)\right) \longrightarrow C^{r-1}\left(\Omega, \mathrm{Cl}\left(V_{n, n}\right)\right) .
$$


Let $u$ be a function with value in $\operatorname{Cl}\left(V_{n, n}\right)$, defined in $\Omega$, and the operator $D$ acts on the function $u$ from the left and from the right, which is being governed by the following rule:

$$
\begin{aligned}
& D[u]=\sum_{k=1}^{n} \sum_{A} e_{k} e_{A} \frac{\partial u_{A}}{\partial x_{k}}, \\
& {[u] D=\sum_{k=1}^{n} \sum_{A} e_{A} e_{k} \frac{\partial u_{A}}{\partial x_{k}} .}
\end{aligned}
$$

Definition 1. A compact surface $\Gamma$ is called Lyapunov surface with Hölder exponent $\alpha$, if the following conditions are satisfied.

(i) At each point $x \in \Gamma$ there is a tangential space.

(ii) There exists a number $r$, such that for any point $x \in$ $\Gamma$ the set $\Gamma \cap B_{r}(x)$ (Lyapunov ball) is connected and parallel lines to the outer normal $\alpha(x)$ intersect at not more than one point.

(iii) The normal $\alpha(x)$ is Hölder continuous on $\Gamma$; that is, there are constants $C>0$ and $0<\alpha \leq 1$ such that for $x, y \in \Gamma$

$$
|\alpha(x)-\alpha(y)| \leq C|x-y|^{\alpha} .
$$

Let $\Omega$ be an open nonempty subset of $\mathscr{R}^{n}$ with a Lyapunov boundary, $u(x)=\sum_{A} e_{A} u_{A}(x)$, where $u_{A}(x)$ are real functions; $u(x)$ is called a Hölder continuous function on $\bar{\Omega}$ if the following condition is satisfied:

$$
\begin{aligned}
& \left|u\left(x_{1}\right)-u\left(x_{2}\right)\right| \\
& \quad=\left[\sum_{A}\left|u_{A}\left(x_{1}\right)-u_{A}\left(x_{2}\right)\right|^{2}\right]^{1 / 2} \leq C\left|x_{1}-x_{2}\right|^{\alpha},
\end{aligned}
$$

where for any $x_{1}, x_{2} \in \bar{\Omega}, x_{1} \neq x_{2}, 0<\alpha \leq 1, C$ is a positive constant independent of $x_{1}, x_{2}$.

Let $H^{\alpha}\left(\partial \Omega, \mathrm{Cl}\left(V_{n, n}\right)\right)$ denote the set of Hölder continuous functions with values in $\mathrm{Cl}\left(V_{n, n}\right)$ on $\partial \Omega$ (the Hölder exponent is $\alpha, 0<\alpha<1)$. We denote the norm in $H^{\alpha}\left(\partial \Omega, \mathrm{Cl}\left(V_{n, n}\right)\right)$ as

$$
\|u\|_{(\alpha, \partial \Omega)}=\|u\|_{\infty}+\|u\|_{\alpha},
$$

where

$$
\begin{aligned}
& \|u\|_{\infty}:=\sup _{x \in \partial \Omega}|u(x)|, \\
& \|u\|_{\alpha}:=\sup _{\substack{x, x_{2} \in \partial \Omega \\
x_{1} \neq x_{2}}} \frac{\left|u\left(x_{1}\right)-u\left(x_{2}\right)\right|}{\left|x_{1}-x_{2}\right|^{\alpha}} .
\end{aligned}
$$

Lemma 2. The Hölder space $H^{\alpha}\left(\partial \Omega, C l\left(V_{n, n}\right)\right)$ is a Banach space with norm (7). by

Denote the fundamental solutions of $D^{j}(j=1,2, \ldots, 6)$

$$
\begin{aligned}
& H_{1}(x)=\frac{1}{\omega_{n}} \frac{\mathbf{x}}{\rho^{n}(x)}, \\
& H_{2}(x)=\frac{1}{2-n} \frac{1}{\omega_{n}} \frac{1}{\rho^{n-2}(x)}, \\
& H_{3}(x)=\frac{1}{2(2-n)} \frac{1}{\omega_{n}} \frac{\mathbf{x}}{\rho^{n-2}(x)}, \\
& H_{4}(x)=\frac{1}{2(2-n)(4-n)} \frac{1}{\omega_{n}} \frac{1}{\rho^{n-4}(x)}, \\
& H_{5}(x)=\frac{1}{8(2-n)(4-n)} \frac{1}{\omega_{n}} \frac{\mathbf{x}}{\rho^{n-4}(x)}, \\
& H_{6}(x)=\frac{1}{8(2-n)(4-n)(6-n)} \frac{1}{\omega_{n}} \frac{1}{\rho^{n-6}(x)},
\end{aligned}
$$

where $\rho(x)=\left(\sum_{i=1}^{n} x_{i}^{2}\right)^{1 / 2}$ and $\omega_{n}$ denotes the area of the unit sphere in $\mathscr{R}^{n}(n \geq 3, n \neq 4, n \neq 6)$.

We will introduce the following operators:

$$
\left(F_{\partial \Omega} u\right)(x)=\int_{\partial \Omega} 2 H_{1}(y-x) d \sigma_{y} u(y), \quad x \in \mathscr{R}^{n} \backslash \partial \Omega,
$$

$$
\left(S_{\partial \Omega} u\right)(x)=\int_{\partial \Omega} 2 H_{1}(y-x) d \sigma_{y} u(y), \quad x \in \partial \Omega,
$$

where $u \in H^{\alpha}\left(\partial \Omega, \operatorname{Cl}\left(V_{n, n}\right)\right)$.

Lemma 3 (see [5]). The integral operator $S_{\partial \Omega}$ in (11) is a bounded linear operator mapping the function space $H^{\alpha}(\partial \Omega$, $\left.\mathrm{Cl}\left(V_{n, n}\right)\right)$ into itself; that is, there exists a positive constant $M$ such that, for all $u \in H^{\alpha}\left(\partial \Omega ; C l\left(V_{n, n}\right)\right)$,

$$
\left\|S_{\partial \Omega} u\right\|_{(\alpha, \partial \Omega)} \leq M\|u\|_{\left(\alpha, C l\left(V_{n, n}\right)\right.} .
$$

\section{Some Properties for Triharmonic Functions}

Theorem 4 (Gauss-mean value formula for triharmonic functions, see $[6,13])$. Suppose $\Delta^{3}[u]=0$ in $\mathscr{R}^{n}$; then, for any $x \in \mathscr{R}^{n}$,

$$
\begin{aligned}
u(x)= & \frac{1}{\omega_{n} R^{n-1}} \int_{\partial B(x, R)} u(y) d S \\
& -\frac{R^{2}}{2 n} \Delta[u](x)-\frac{R^{4}}{8 n(n+2)} \Delta^{2}[u](x),
\end{aligned}
$$

or

$$
\begin{aligned}
u(x)= & \frac{n}{\omega_{n} R^{n}} \int_{B(x, R)} u(y) d V \\
& -\frac{R^{2}}{2(n+2)} \Delta[u](x)-\frac{R^{4}}{8(n+2)(n+4)} \Delta^{2}[u](x),
\end{aligned}
$$

where $\omega_{n}$ denotes the area of the unit sphere in $\mathscr{R}^{n}$. 
Corollary 5. Suppose $\Delta^{3}[u]=0$ in $\mathscr{R}^{n}$ and $|u(x)|=O(|x|)$ $(|x| \rightarrow \infty)$; then $\Delta[u]=0$ and $\Delta^{2}[u]=0$ in $\mathscr{R}^{n}$.

Corollary 6. Suppose $\Delta^{3}[u]=0$ in $\mathscr{R}^{n}$ and $u(x)$ is bounded in $\mathscr{R}^{n}$; then $u(x) \equiv C$.

Theorem 7. Suppose $\Delta^{3}[u]=0$ in $\mathscr{R}^{n} \backslash \partial \Omega$, and for $x \in \partial \Omega$, $u \in C^{6}\left(\mathscr{R}^{n} \backslash \partial \Omega, C l\left(V_{n, n}\right)\right),[u]^{+}(x)=[u]^{-}(x) \in C^{5}(\partial \Omega$, $\left.\mathrm{Cl}\left(V_{n, n}\right)\right)$, and, moreover, $D^{j}[u]^{+}(x)=D^{j}[u]^{-}(x) \in H^{\alpha_{j}}(\partial \Omega$, $\left.C l\left(V_{n, n}\right)\right), 0<\alpha_{j} \leq 1$, where $j=0,1, \ldots, 5$. Then $\Delta^{3}[u]=0$ in $\mathscr{R}^{n}$.

Theorem 8. Let $u \in C^{6}\left(\Omega^{-}, C l\left(V_{n, n}\right)\right) \cap C^{5}\left(\overline{\Omega^{-}}, C l\left(V_{n, n}\right)\right)$, $\Delta^{3}[u]=0$ in $\Omega^{-}, D^{j}[u](x) \in H^{\alpha_{j}}\left(\partial \Omega, C l\left(V_{n, n}\right)\right), 0<\alpha_{j} \leq 1$ $(j=0,1, \ldots, 5)$, and $|u(x)|=O(1)(|x| \rightarrow \infty)$; then for $x \in \Omega^{-}$

$$
u(x)=\sum_{j=0}^{5}(-1)^{j+1} \int_{\partial \Omega} H_{j+1}(y-x) d \sigma_{y} D^{j}[u](y)+C,
$$

where $H_{j}(y-x)$ is as in (9) and $C$ is a constant.

Proof. For $y \in \partial \Omega$, denote $D^{j}[u](y)=-\Psi_{j}(y),(j=0,1$, $\ldots, 5)$. For $x \in \mathscr{R}^{n} \backslash \partial \Omega$, denoting

$$
\begin{gathered}
\Theta(x)=\sum_{j=0}^{5}(-1)^{j} \int_{\partial \Omega} H_{j+1}(y-x) d \sigma_{y} \Psi_{j}(y), \\
u^{*}(x)= \begin{cases}-\Theta(x), & x \in \Omega^{+} \\
u(x)-\Theta(x), & x \in \Omega^{-},\end{cases}
\end{gathered}
$$

then $\Delta^{3}\left[u^{*}\right]=0$ in $\mathscr{R}^{n} \backslash \partial \Omega$. By using Plemelj formula, combining with weak singularity of $H_{j}(x)(j \geq 2)$, we obtain the following:

$$
D^{j}\left[u^{*}\right]^{+}(x)=D^{j}\left[u^{*}\right]^{-}(x) \in H^{\alpha_{j}^{\prime}}\left(\partial \Omega, \mathrm{Cl}\left(V_{n, n}\right)\right),
$$

where $0<\alpha_{j}^{\prime} \leq 1(j=0,1, \ldots, 5)$. By using Theorem 7 , we have $\Delta^{3}\left[u^{*}\right]=0$ in $\mathscr{R}^{n}$. It is clear that we have $\left|u^{*}(x)\right|=$ $O(1)(|x| \rightarrow \infty)$. In view of Corollary 6 , then the results follow.

Corollary 9. Let $u \in C^{6}\left(\Omega^{-}, C l\left(V_{n, n}\right)\right) \cap C^{5}\left(\overline{\Omega^{-}}, C l\left(V_{n, n}\right)\right)$, $\Delta^{3}[u]=0$ in $\Omega^{-}, D^{j}[u](x) \in H^{\alpha_{j}}\left(\partial \Omega, C l\left(V_{n, n}\right)\right), 0<\alpha_{j} \leq 1$ $(j=0,1, \ldots, 5)$, and $|u(x)|=O(1)(|x| \rightarrow \infty)$, then

$$
D^{j}[u](\infty)=0, \quad j=1, \ldots, 5,
$$

Remark 10. When the condition $|u(x)|=O(1)(|x| \rightarrow \infty)$ in Corollary 9 is replaced by $u(\infty)=0$, then the results in Corollary 9 are still valid.

\section{Riemann Boundary Value Problem for Triharmonic Functions}

In this section, we will consider the Riemann boundary value problem (1); the explicit expression of the solution is given.
Theorem 11. The Riemann boundary value problem (1) is solvable and the solution can be written as

$$
u(x)= \begin{cases}\sum_{i=1}^{6} \Psi_{i}(x)+C, & x \in \Omega, \\ \sum_{i=1}^{6} \Psi_{i}(x)+C A^{-1}, & x \in \Omega^{-},\end{cases}
$$

where

$$
\Psi_{1}(x)= \begin{cases}-\int_{\partial \Omega} H_{6}(y-x) d \sigma_{y} f_{5}(y), & x \in \Omega^{+} \\ -\int_{\partial \Omega} H_{6}(y-x) d \sigma_{y} f_{5}(y) A_{5}^{-1}, & x \in \Omega^{-}\end{cases}
$$

$$
\Psi_{2}(x)= \begin{cases}\int_{\partial \Omega} H_{5}(y-x) d \sigma_{y} \tilde{f}_{4}(y), & x \in \Omega^{+} \\ \int_{\partial \Omega} H_{5}(y-x) d \sigma_{y} \widetilde{f}_{4}(y) A_{4}^{-1}, & x \in \Omega^{-}\end{cases}
$$

$$
\Psi_{3}(x)= \begin{cases}-\int_{\partial \Omega} H_{4}(y-x) d \sigma_{y} \widetilde{f}_{3}(y), & x \in \Omega^{+} \\ -\int_{\partial \Omega} H_{4}(y-x) d \sigma_{y} \widetilde{f}_{3}(y) A_{3}^{-1}, & x \in \Omega^{-}\end{cases}
$$

$$
\Psi_{4}(x)= \begin{cases}\int_{\partial \Omega} H_{3}(y-x) d \sigma_{y} \tilde{f}_{2}(y), & x \in \Omega^{+} \\ \int_{\partial \Omega} H_{3}(y-x) d \sigma_{y} \tilde{f}_{2}(y) A_{2}^{-1}, & x \in \Omega^{-}\end{cases}
$$

$$
\Psi_{5}(x)= \begin{cases}-\int_{\partial \Omega} H_{2}(y-x) d \sigma_{y} \widetilde{f}_{1}(y), & x \in \Omega^{+} \\ -\int_{\partial \Omega} H_{2}(y-x) d \sigma_{y} \widetilde{f}_{1}(y) A_{1}^{-1}, & x \in \Omega^{-}\end{cases}
$$

$$
\Psi_{6}(x)= \begin{cases}\int_{\partial \Omega} H_{1}(y-x) d \sigma_{y} \tilde{g}(y), & x \in \Omega^{+} \\ \int_{\partial \Omega} H_{1}(y-x) d \sigma_{y} \tilde{g}(y) A^{-1}, & x \in \Omega^{-}\end{cases}
$$

$$
\tilde{f}_{4}(x)=f_{4}(x)-\int_{\partial \Omega} H_{2}(y-x) d \sigma_{y} f_{5}(y)\left(-1+A_{5}^{-1} A_{4}\right),
$$

$$
\begin{aligned}
\tilde{f}_{3}(x)= & f_{3}(x)+\int_{\partial \Omega} H_{3}(y-x) d \sigma_{y} f_{5}(y)\left(-1+A_{5}^{-1} A_{3}\right) \\
& -\int_{\partial \Omega} H_{2}(y-x) d \sigma_{y} \tilde{f}_{4}(y)\left(-1+A_{4}^{-1} A_{3}\right),
\end{aligned}
$$




$$
\begin{aligned}
\tilde{f}_{2}(x)= & f_{2}(x)-\int_{\partial \Omega} H_{4}(y-x) d \sigma_{y} f_{5}(y)\left(-1+A_{5}^{-1} A_{2}\right) \\
& +\int_{\partial \Omega} H_{3}(y-x) d \sigma_{y} \widetilde{f}_{4}(y)\left(-1+A_{4}^{-1} A_{2}\right) \\
& -\int_{\partial \Omega} H_{2}(y-x) d \sigma_{y} \widetilde{f}_{3}(y)\left(-1+A_{3}^{-1} A_{2}\right),
\end{aligned}
$$$$
x \in \partial \Omega \text {, }
$$

$$
\begin{aligned}
\tilde{f}_{1}(x)= & f_{1}(x) \\
& +\int_{\partial \Omega} H_{5}(y-x) d \sigma_{y} f_{5}(y)\left(-1+A_{5}^{-1} A_{1}\right) \\
& -\int_{\partial \Omega} H_{4}(y-x) d \sigma_{y} \tilde{f}_{4}(y)\left(-1+A_{4}^{-1} A_{1}\right) \\
& +\int_{\partial \Omega} H_{3}(y-x) d \sigma_{y} \tilde{f}_{3}(y)\left(-1+A_{3}^{-1} A_{1}\right) \\
& -\int_{\partial \Omega} H_{2}(y-x) d \sigma_{y} \tilde{f}_{2}(y)\left(-1+A_{2}^{-1} A_{1}\right),
\end{aligned}
$$$$
x \in \partial \Omega \text {, }
$$

$$
\begin{aligned}
\widetilde{g}(x)= & g(x) \\
& -\int_{\partial \Omega} H_{6}(y-x) d \sigma_{y} f_{5}(y)\left(-1+A_{5}^{-1} A\right) \\
& +\int_{\partial \Omega} H_{5}(y-x) d \sigma_{y} \widetilde{f}_{4}(y)\left(-1+A_{4}^{-1} A\right) \\
& -\int_{\partial \Omega} H_{4}(y-x) d \sigma_{y} \tilde{f}_{3}(y)\left(-1+A_{3}^{-1} A\right) \\
& +\int_{\partial \Omega} H_{3}(y-x) d \sigma_{y} \tilde{f}_{2}(y)\left(-1+A_{2}^{-1} A\right) \\
& -\int_{\partial \Omega} H_{2}(y-x) d \sigma_{y} \tilde{f}_{1}(y)\left(-1+A_{1}^{-1} A\right),
\end{aligned}
$$$$
x \in \partial \Omega \text {. }
$$

\section{Existence of Solutions for \\ Riemann Boundary Value Problem for Triharmonic Functions}

Theorem 12. Suppose $G(x) \in H^{\alpha}\left(\partial \Omega, C l\left(V_{n, n}\right)\right) 0<\alpha<1$ and $G(x)$ satisfies the following condition:

$$
2^{n-2}\|1-G(x)\|_{(\alpha, \partial \Omega)}(M+1)<1
$$

where $M$ is the positive constant mentioned in Lemma 3. Then (2) admits a unique solution.
Proof. Denoting $w(x)=D^{5}[u](x)$ then $w^{+}(x)=w^{-}(x) A_{5}+$ $f_{5}(x), x \in \partial \Omega$. Moreover, by $D^{5}[u](\infty)=0$. we get the following:

$$
\omega(x)= \begin{cases}\int_{\partial \Omega} H_{1}(y-x) d \sigma_{y} f_{5}(y), & x \in \Omega^{+} \\ \int_{\partial \Omega} H_{1}(y-x) d \sigma_{y} f_{5}(y) A_{5}^{-1}, & x \in \Omega^{-},\end{cases}
$$

With $\Psi_{1}(x)$ being as in (20), it is easy to check that

$$
D^{5}\left(u-\Psi_{1}\right)(x)=0, \quad x \in \mathscr{R}^{n} \backslash \partial \Omega .
$$

Denoting $\Delta^{2} u(x)-\Delta^{2} \Psi_{1}(x):=\varphi_{1}(x), x \in \mathscr{R}^{n} \backslash \partial \Omega$, and using $\left(\Delta^{2} u\right)^{+}(x)=\left(\Delta^{2} u\right)^{-}(x) A_{4}+f_{4}(x), x \in \partial \Omega$, we conclude that

$$
\varphi_{1}^{+}(x)=\varphi_{1}^{-}(x) A_{4}+\widetilde{f}_{4}(x), \quad x \in \partial \Omega,
$$

where $\tilde{f}_{4}(x) \in H^{\tilde{\beta}}\left(\partial \Omega, \operatorname{Cl}\left(V_{n, n}\right)\right), 0<\widetilde{\beta} \leq 1$ being as in (26). By Corollary 9 , it is clear that $\varphi_{1}(\infty)=0$, and we then get the following representation formula:

$$
\varphi_{1}(x)= \begin{cases}\int_{\partial \Omega} H_{1}(y-x) d \sigma_{y} \tilde{f}_{4}(y), & x \in \Omega^{+} \\ \int_{\partial \Omega} H_{1}(y-x) d \sigma_{y} \tilde{f}_{4}(y) A_{4}^{-1}, & x \in \Omega^{-} .\end{cases}
$$

Analogously, we find with $\Psi_{2}(x)$ from (21) that $\Delta^{2} u(x)-$ $\Delta^{2} \Psi_{1}(x)-\Delta^{2} \Psi_{2}(x)=0, x \in \mathscr{R}^{n} \backslash \partial \Omega$. Denote

$$
\begin{array}{r}
D^{3} u(x)-D^{3} \Psi_{1}(x)-D^{3} \Psi_{2}(x):=\varphi_{2}(x), \\
x \in \mathscr{R}^{n} \backslash \partial \Omega .
\end{array}
$$

Using the condition $\left(D^{3} u\right)^{+}(x)=\left(D^{3} u\right)^{-}(x) A_{3}+f_{3}(x), x \in$ $\partial \Omega$, we obtain that

$$
\varphi_{2}^{+}(x)=\varphi_{2}^{-}(x) A_{3}+\tilde{f}_{3}(x), \quad x \in \partial \Omega,
$$

where $\widetilde{f}_{3}(x) \in H^{\widetilde{\beta}}\left(\partial \Omega, \mathrm{Cl}\left(V_{n, n}\right)\right), 0<\widetilde{\beta} \leq 1$ being as in (27). By Corollary 9 , it is clear that $\varphi_{2}(\infty)=0$, and we then get the following representation formula:

$$
\varphi_{2}(x)= \begin{cases}\int_{\partial \Omega} H_{1}(y-x) d \sigma_{y} \tilde{f}_{3}(y), & x \in \Omega^{+} \\ \int_{\partial \Omega} H_{1}(y-x) d \sigma_{y} \widetilde{f}_{3}(y) A_{3}^{-1}, & x \in \Omega^{-} .\end{cases}
$$

Here $\Psi_{3}(x)$ being as in (22), then we have $D^{3} u(x)-$ $D^{3} \Psi_{1}(x)-D^{3} \Psi_{2}(x)-D^{3} \Psi_{3}(x)=0, x \in \mathscr{R}^{n} \backslash \partial \Omega$. We denote

$$
\Delta u(x)-\Delta \Psi_{1}(x)-\Delta \Psi_{2}(x)-\Delta \Psi_{3}(x):=\varphi_{3}(x)
$$

and use the condition $(\Delta u)^{+}(x)=(\Delta u)^{-}(x) A_{2}+f_{2}(x), x \in$ $\partial \Omega$. We conclude that

$$
\varphi_{3}^{+}(x)=\varphi_{3}^{-}(x) A_{2}+\widetilde{f}_{2}(x), \quad x \in \partial \Omega,
$$


where $\tilde{f}_{2}(x) \in H^{\widetilde{\beta}}\left(\partial \Omega, \operatorname{Cl}\left(V_{n, n}\right)\right), 0<\widetilde{\beta} \leq 1$ being as in (28). Corollary 9 ensures that $\varphi_{3}(\infty)=0$; then

$$
\varphi_{3}(x)= \begin{cases}\int_{\partial \Omega} H_{1}(y-x) d \sigma_{y} \tilde{f}_{2}(y), & x \in \Omega^{+} \\ \int_{\partial \Omega} H_{1}(y-x) d \sigma_{y} \tilde{f}_{2}(y) A_{2}^{-1}, & x \in \Omega^{-} .\end{cases}
$$

Use the same way again, $\Psi_{4}(x)$ being as in (23), that $\Delta u(x)-$ $\Delta \Psi_{1}(x)-\Delta \Psi_{2}(x)-\Delta \Psi_{3}(x)-\Delta \Psi_{4}(x)=0, x \in \mathscr{R}^{n} \backslash \partial \Omega$. Denoting

$$
D u(x)-\sum_{i=1}^{4} D \Psi_{i}(x):=\varphi_{4}(x), \quad x \in \mathscr{R}^{n} \backslash \partial \Omega
$$

and using $(D u)^{+}(x)=(D u)^{-}(x) A_{1}+f_{1}(x), x \in \partial \Omega$, we get

$$
\varphi_{4}^{+}(x)=\varphi_{4}^{-}(x) A_{1}+\tilde{f}_{1}(x), \quad x \in \partial \Omega,
$$

where $\tilde{f}_{1}(x) \in H^{\tilde{\beta}}\left(\partial \Omega, \operatorname{Cl}\left(V_{n, n}\right)\right), 0<\tilde{\beta} \leq 1$ being taken from (29). By Corollary 9, it is clear that $\varphi_{4}(\infty)=0$, and we then get the following representation formula:

$$
\varphi_{4}(x)= \begin{cases}\int_{\partial \Omega} H_{1}(y-x) d \sigma_{y} \tilde{f}_{1}(y), & x \in \Omega^{+} \\ \int_{\partial \Omega} H_{1}(y-x) d \sigma_{y} \tilde{f}_{1}(y) A_{1}^{-1}, & x \in \Omega^{-} .\end{cases}
$$

Finally, we use $\Psi_{5}(x)$ as defined in (24) and get $D u(x)$ $\sum_{i=1}^{4} D \Psi_{i}(x)=0, x \in \mathscr{R}^{n} \backslash \partial \Omega$. Define

$$
u(x)-\sum_{i=1}^{5} \Psi_{i}(x):=\varphi_{5}(x), \quad x \in \mathscr{R}^{n} \backslash \partial \Omega .
$$

Working with the condition $u^{+}(x)=u^{-}(x) G(x)+g(x)$ we arrive at

$$
\varphi_{5}^{+}(x)=\varphi_{5}^{-}(x) G(x)+\widetilde{g}(x), \quad x \in \partial \Omega,
$$

where $\tilde{g}(x) \in H^{\tilde{\beta}}\left(\partial \Omega, \operatorname{Cl}\left(V_{n, n}\right)\right), 0<\tilde{\beta} \leq 1$ being taken from (30). It is clear that $\varphi_{5}(\infty)=0$. We obtain that

$$
\begin{aligned}
D \varphi_{5} & =0, \quad \mathscr{R}^{n} \backslash \partial \Omega \\
\varphi_{5}^{+}(x) & =\varphi_{5}^{-}(x) G(x)+\widetilde{g}(x), \quad x \in \partial \Omega \\
\varphi_{5}(\infty) & =0 .
\end{aligned}
$$

We only need to consider the existence of solutions to (47). The solution to this problem may be written in the form

$$
\varphi_{5}(x)=\int_{\partial \Omega} H_{1}(y-x) d \sigma_{y} \varphi_{6}(y),
$$

where $\varphi_{6}(y)$ is a Hölder continuous function to be determined on $\partial \Omega$. Then, by using Plemelj formula, (47) can be reduced to an equivalent singular integral equation for $\varphi_{6}$,

$$
\begin{aligned}
& \varphi_{6}(x) \\
& =\left[\frac{\varphi_{6}(x)}{2}-\int_{\partial \Omega} H_{1}(y-x) d \sigma_{y} \varphi_{6}(y)\right](1-G(x))+\tilde{g}(x),
\end{aligned}
$$

Letting $T$ denote the integral operator defined by the right hand side of (49), we get

$$
\left(T \varphi_{6}\right)(x)=\left[\varphi_{6}(x)-\left(S_{\partial \Omega} \varphi_{6}\right)(x)\right] \frac{(1-G(x))}{2}+\widetilde{g(x)} .
$$

For any $\omega_{1}, \omega_{2} \in H^{\alpha}\left(\partial \Omega, \mathrm{Cl}\left(V_{n, n}\right)\right)$, we have

$$
\begin{aligned}
& \left\|T \omega_{1}-T \omega_{2}\right\|_{(\alpha, \partial \Omega)} \\
& \quad \leq \frac{2^{n-1}}{2}\left\|\omega_{1}-\omega_{2}\right\|_{(\alpha, \partial \Omega)}\|1-G\|_{(\alpha, \partial \Omega)}(1+M) .
\end{aligned}
$$

Under the condition (31), the integral operator $T$ is a contraction operator mapping the Banach space $H^{\alpha}\left(\partial \Omega, \mathrm{Cl}\left(V_{n, n}\right)\right)$ into itself, which has a unique fixed point for the operator $T$. Thus, there exists a unique solution to (47). The proof is done.

\section{Conflict of Interests}

The author declares that there is no conflict of interests regarding the publication of this paper.

\section{Acknowledgments}

This paper is supported by National Natural Science Foundation of China (11271175), the AMEP, and DYSP of Linyi University.

\section{References}

[1] J. K. Lu, Boundary Value Problems for Analytic Functions, World Scientific Publishing, Singapore, 1993.

[2] N. I. Muskhelishvilli, Singulyarnye Integralnye Uravneniya, Moscow, Russia, 1968.

[3] Y. Gong and J. Du, "A kind of Riemann and Hilbert boundary value problem for left monogenic functions in $R^{m}(m \geq 2)$," Complex Variables, Theory and Application, vol. 49, no. 5, pp. 303-318, 2004.

[4] Z. Zhang and K. Gürlebeck, "Some Riemann boundary value problems in Clifford analysis (I)," Complex Variables and Elliptic Equations, vol. 58, no. 7, pp. 991-1003, 2013.

[5] Z. Y. Xu, "On linear and nonlinear Riemann-Hilbert problems for regular function with values in a Clifford algebra," Chinese Annals of Mathematics B, vol. 11, no. 3, pp. 349-358, 1990.

[6] L. Gu, J. Du, and Z. Zhang, "Riemann boundary value problems for triharmonic functions in Clifford analysis," Advances in Applied Clifford Algebras, vol. 23, no. 1, pp. 77-103, 2013.

[7] T. S. Kalmenov and D. B. Koshanov, "Representation for the Green's function of the Dirichlet problem for polyharmonic equations in a ball," Siberian Mathematical Journal, vol. 1, pp. 423-428, 2008.

[8] Z. Zhang, "On $k$-regular functions with values in a universal Clifford algebra," Journal of Mathematical Analysis and Applications, vol. 315, no. 2, pp. 491-505, 2006.

[9] H. Begehr, J. Du, and Z. Zhang, "On higher order CauchyPompeiu formula in CLIfford analysis and its applications," General Mathematics, vol. 11, no. 3-4, pp. 5-26, 2003 (Romanian). 
[10] R. Delanghe, "On regular-analytic functions with values in a Clifford algebra," Mathematische Annalen, vol. 185, pp. 91-111, 1970.

[11] R. Delanghe, "On the singularities of functions with values in a Clifford algebra," Mathematische Annalen, vol. 196, pp. 293-319, 1972.

[12] F. Brackx, R. Delanghe, and F. Sommen, Clifford Analysis, vol. 76 of Research Notes in Mathematics, Pitman, London, UK, 1982.

[13] L. Gu and Z. Zhang, "Gauss-mean value formula for triharmonic functions and its applications in Clifford analysis," Acta Mathematica Scientia B, vol. 33, no. 5, pp. 1419-1430, 2013. 


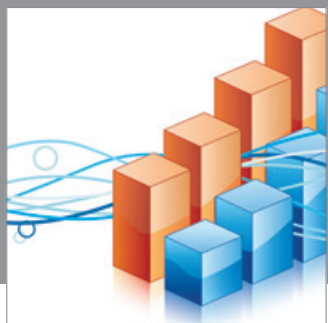

Advances in

Operations Research

mansans

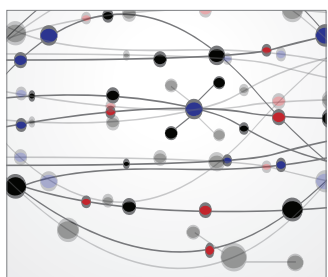

The Scientific World Journal
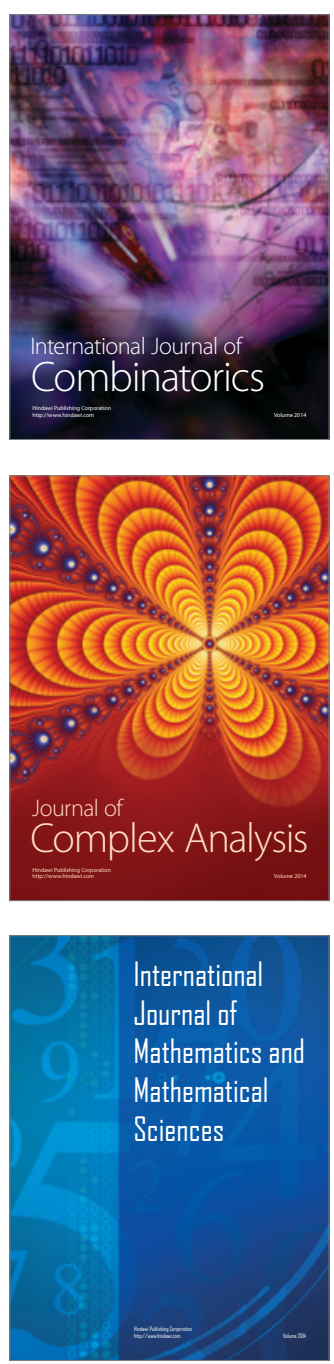
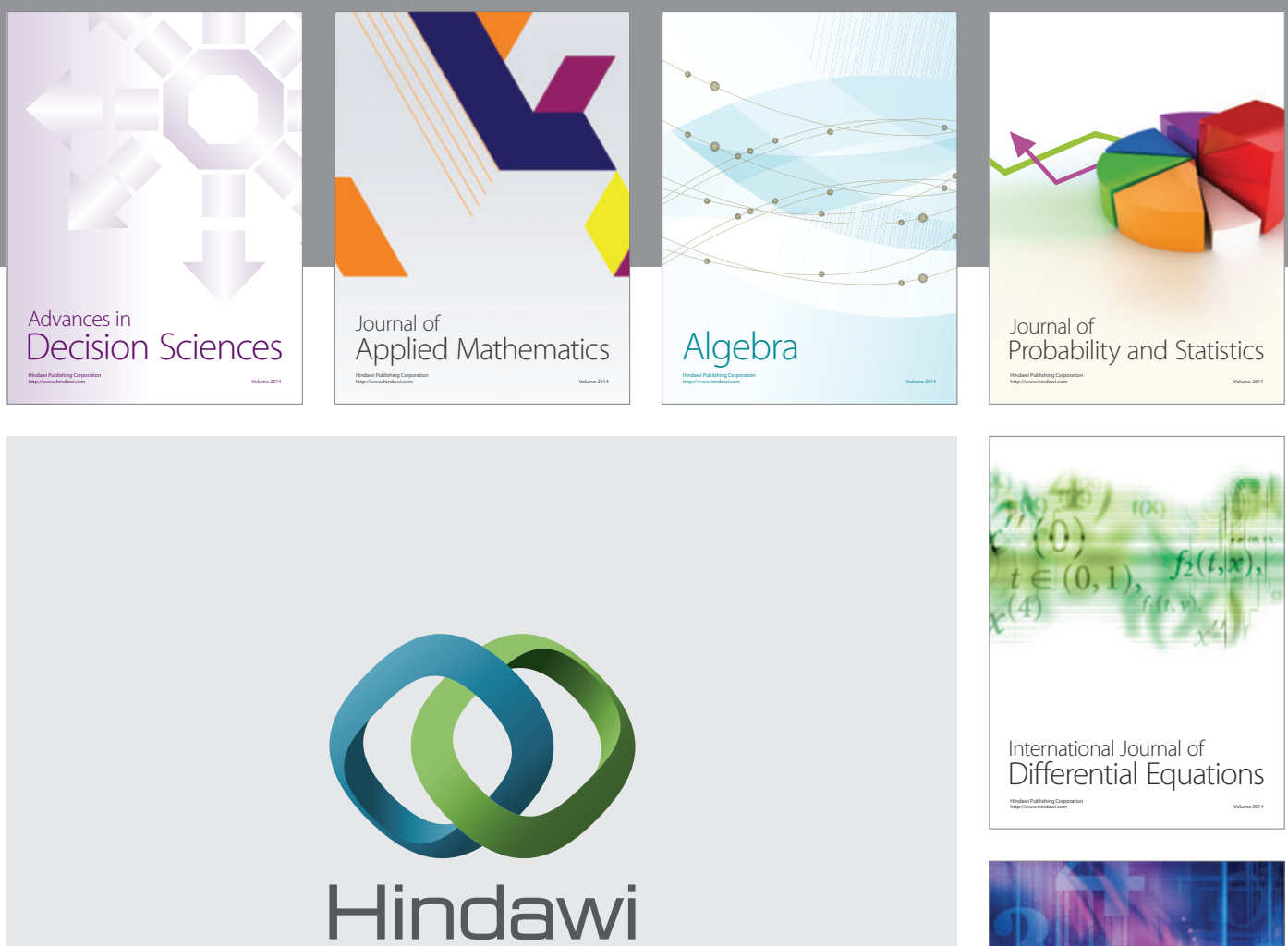

Submit your manuscripts at http://www.hindawi.com
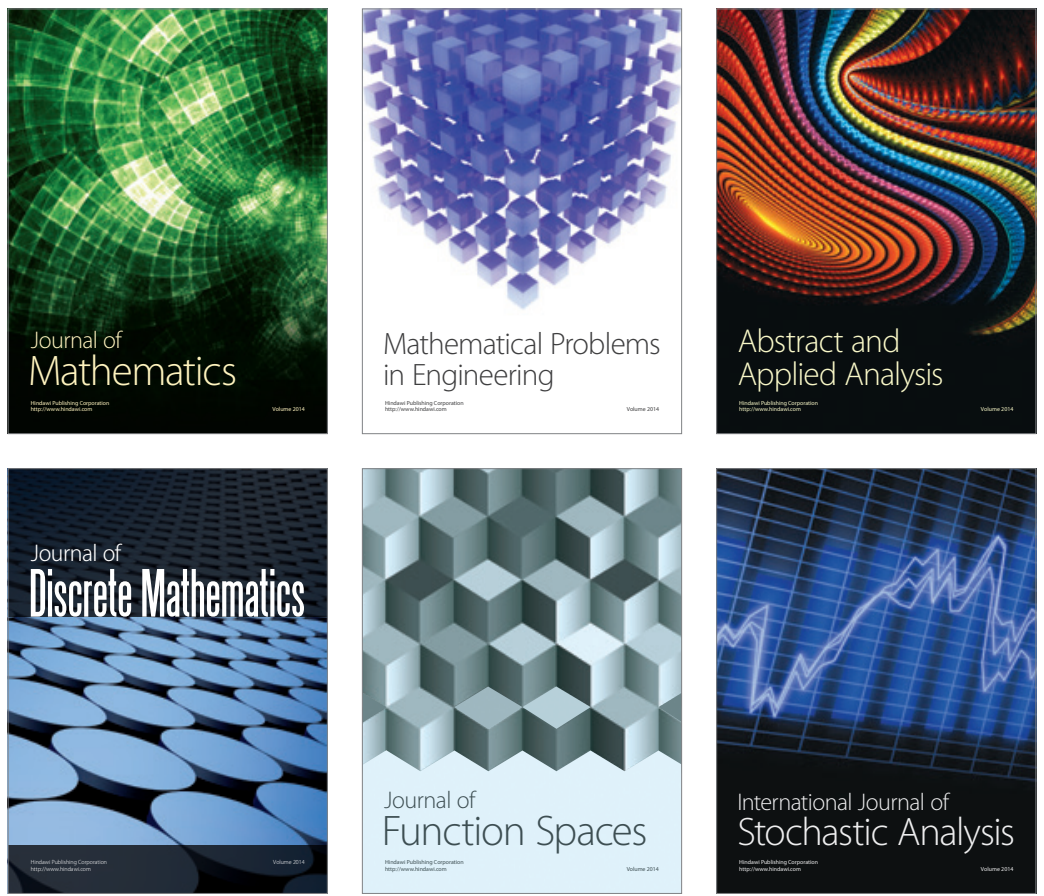

Journal of

Function Spaces

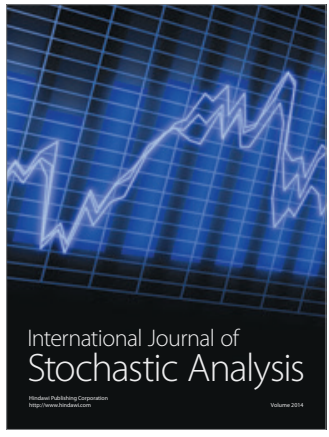

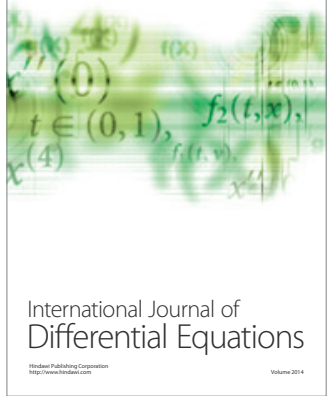
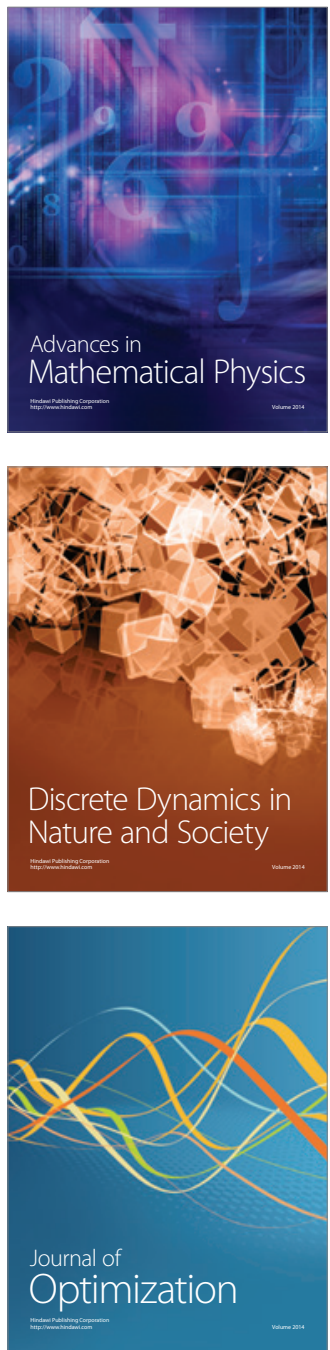